\title{
Exact Solution for Search-and-Rescue Path Planning
}

\author{
Jean Berger, Nassirou Lo, and Martin Noel
}

\begin{abstract}
Discrete search and rescue path planning is known to be hard, and problem-solving techniques proposed so far mainly fail to properly assess optimality gap for practical size problems. A new mixed-integer linear programming (MIP) formulation is proposed to optimally solve the single agent discrete search and rescue (SAR) path planning problem. The approach lies on a compact open-loop SAR with anticipated feedback problem model to efficiently maximize cumulative probability of success in detecting a target. Anticipated feedback information resulting from possible observations outcomes along the path is exploited to update target occupancy beliefs. A network representation is utilized to simplify modeling, facilitate constraint specification and speed-up problem-solving. The proposed MIP approach rapidly yields optimal solutions for realistic problems using parallel processing CPLEX technology, while providing for the first time a robust upper bound on solution quality through Lagrangean integrality constraint relaxation. Fast computation naturally allows extending open-loop modeling to a closed-loop environment to progressively integrate real-time action outcomes as they occur on a rolling time horizon. Comparative performance results clearly show the value of the approach.
\end{abstract}

Index Terms-Search path planning, search and rescue, linear programming.

\section{INTRODUCTION}

Target search and rescue path planning is a top priority life-saving task facing human teams when struggling with man-made and natural disasters. It is a pervasive problem increasingly occurring over a variety of civilian and military domains such as homeland security and emergency management. The basic discrete SAR or optimal searcher path problem involving a stationary target is known to be NP-Hard [1]. SAR may be generally characterized through multiple dimensions and attributes including: one-sided search in which targets are non-responsive toward searcher's actions, two-sided, describing target behaviour diversity (cooperative, non-cooperative or anti-cooperative), stationary Vs. moving target search, discrete Vs. continuous time and space search (efforts indivisibility/divisibility), observation model, static/dynamic as well as open and closed-loop decision models, pursued objectives, target and searcher multiplicity and diversity. Early work on related search problems emerges from search theory, [2], [3]. Search-theoretic approaches mostly relate to the effort (time

Manuscript received October 9, 2012; revised January 18, 2013.

J. Berger is with the Defence Research and Development Canada Valcartier, Quebec City, Canada (e-mail: jean.berger@drdc-rddc.gc.ca).

N. Lo is with the T-OptLogic Ltd., Quebec City Canada (e-mail: nassirou.lo@t-optlogic.com).

M. Noel is with the Téluq, Université du Québec, Quebec City Canada (e-mail: noel.martin@teluq.uqam.ca). spent per visit) allocation decision problem rather than path construction. Based upon a mathematical framework, efforts have increasingly been devoted to algorithmic contributions to handle more complex dynamic problem settings and variants [4]-[7]. In counterpart, many contributions on search path planning may be found in the robotics literature in the area of robot motion planning [8] and, namely, terrain acquisition [9], [10] and coverage path planning [11]-[13]. Robot motion planning explored search path planning, primarily providing constrained shortest path type solutions for coverage problem instances [14], [15]. These studies typically examine uncertain search environment problems with limited prior domain knowledge, involving unknown sparsely distributed static targets and obstacles. Recent taxonomies and comprehensive surveys on target search problems from search theory and artificial intelligence distributed robotic control perspectives may be found in [5], [17]-[19] respectively.

Exact problem-solving methods for sequential decision search problem formulations show computational complexity to scale exponentially. For instance, dynamic programming [5], [7], [19], [20] or tree-based search techniques [21] may satisfactorily work under specific constraints and conditions but ultimately face the curse of dimensionality, showing poor scalability even for moderate size problem. This paved the way to the development of efficient heuristic and approximate methods. But published procedures still deliver approximate solution and mostly fail to provably estimate real performance optimality gap for practical size problems, questioning their real expected relative efficiency.

In this paper, we propose a new exact mixed-integer linear programming formulation to optimally solve the single agent discrete search path planning problem for a stationary object. In the problem model, 'open-loop with anticipated feedback' refers to offline planning, while capturing information resulting from predicted agent observations (projected cell visit action outcome) as opposed to real feedback. Anticipated feedback augments pure open-loop formulations which simply ignore information feedback, while significantly improving solution quality, and mitigating computational complexity limitations traditionally associated with closed-loop problem formulations (e.g. dynamic programming, and partially observable Markov decision processes). In that setting, the open-loop with anticipated feedback information (observations) decision model involves an agent (the searcher) with imperfect sensing capability (but false alarm -free) searching an area (grid) to maximize cumulative probability of success in detecting a target, given a time horizon and prior cell occupancy probability distribution. The model takes advantage of anticipated feedback information resulting from observations outcomes along the path to update target occupancy beliefs and make better decisions. A network flow representation significantly 
reduces modeling complexity (e.g. constraint specification) as well as implementation and computational costs. The new decision model relies on an abstract network representation, coupled to a parallel computing capability (e.g. using the CPLEX solver [22], [23]) to gain additional speed-up. The novelty lies in a new exact linear model, and the fast computation of near optimal solutions of practical size problems, providing for the first time a tight upper bound on solution quality through Lagrangean programming relaxation. The computable upper bound constitutes an objective measure to fairly estimate and compare performance gap against various techniques. Computational results prove the proposed approach very efficient and to outperform alternate techniques over a random sample of problem instances. Small computational run-time naturally enables open-loop model (with anticipated feedback) extension to a closed-loop formulation in which action outcomes from the previous episode may be explicitly incorporated in real-time to update target occupancy belief distribution.

The remainder of the paper is structured as follows. Section II first introduces problem definition, describing the main characteristics of the open-loop search path planning problem with anticipated feedback. Then the main solution concept for the problem is presented in Section III. Section IV reports and discusses computational results while comparing the value of the proposed method to an alternate myopic problem-solving technique. Finally, a conclusion is given in Section $\mathrm{V}$.

\section{PRoBlem DEFINITION}

\section{A. Description}

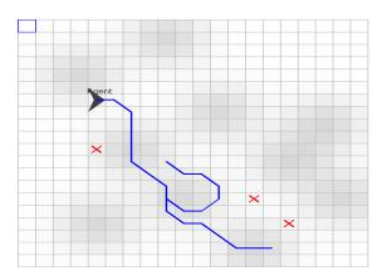

Fig. 1. Uncertainty grid /cognitive map at time step t. Beliefs are displayed through multi-level shaded cell areas. Projected agent plans are represented as possible paths.

The discrete centralized search and rescue path planning problem involves a stand-off sensor agent searching a stationary target in a bounded environment over a given time horizon. From a search and rescue mission perspective, the goal consists in maximizing the cumulative probability of success in detecting a target within a given region. Modeled as a grid, the search region defines a two-dimensional cellular area forming a set of cells $N$, populated by a single stationary target, assumed to occupy a single cell. Target location is unknown. Based on domain knowledge, a prior target location probability density distribution defines individual cell occupancy, characterizing a grid cognitive map. Target occupancy probabilities over grid cells sum to one. Should the target be located outside the search areas of interest, a special inaccessible, and invisible virtual cell would simply be added. The cognitive map or uncertainty grid is a knowledge base capturing local environment state representation, reflecting target occupancy belief distribution, agent position and orientation. A typical cognitive map at a given point in time is illustrated in Fig. 1.

Cell visit time specifying episode duration is assumed to be constant. Vehicles are assumed to fly at slightly different altitudes to avoid colliding with one other. A search path solution consists in constructing an agent path plan to maximize target detection.

\section{B. Path Planning}

A centralized decision-making process episodically makes an agent's search path planning decision based on agent's position (cell location), and specific orientation $\{N, S, E, W$, $N E, S E, S W, N W\}$. In Fig. 2 decisions for a 3-move agent are limited to three possible directions with respect to its current heading, namely, ahead, right or left. These moves account for physical acceleration associated to agent motion.

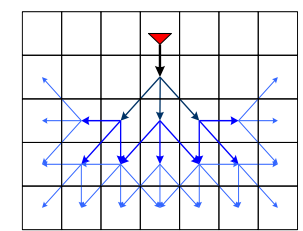

Fig. 2. Agent's region of interest displayed as forward move projection span (possible paths), for a 3-moving direction agent over a 3-step time horizon.

\section{Cumulative Probability of Success}

In the proposed open-loop SAR model, the probability to successfully detect the target resulting from an agent path solution execution on the grid is defined as the sum over cells of the product of the probability of detection reflected from cell visits and target cell occupancy belief dictated by the cognitive map (grid) [5], [24]. Cumulative probability of success $(C P O S)$ for a path solution (sequence of cell visits) over a time horizon $T$ can then be expressed as follows:

$$
C P O S=\sum_{c \in N} \sum_{t} \operatorname{pos}_{c t}=\sum_{c \in N} \sum_{t} p_{c c} p_{c t}
$$

where $\operatorname{pos}_{c t}$ represents the probability of successfully detecting the target during a visit in cell $c$ over the period $t$ given it has not been detected during earlier visits. $p_{c t}$ refers to the probability/belief of cell target occupancy during time interval $t$ which incorporates "anticipated" information feedback that would result from past visits. As for $p_{c c}$, it is the probability on a specific agent visit $c$ to correctly detect the target in cell $c$ given that the target is present in cell $c . p_{c c}$ depends on cell $c$. The agent sensor is assumed to be false-alarm free, meaning that a vacant cell visit corresponds to a negative observation by the sensing agent. Conversely, based on this assumption, a positive observation confirms that the target is found and that the search task is terminated. In the current setting, sensor range defining visibility or footprint (observable cells given current sensor position) is limited to the cell being searched.

\section{MiXed-InTEger Linear PROGRAmMing ModeL FORMULATION}

\section{A. Network Representation}

A network representation is used to simplify modeling and 
constraint specification as well as problem-solving, as it eliminates the need to explicitly capture all constraints. These include maximum path length or deadline, admissible/legal move, and disconnected subtours elimination which may significantly impact run-time when handled explicitly.

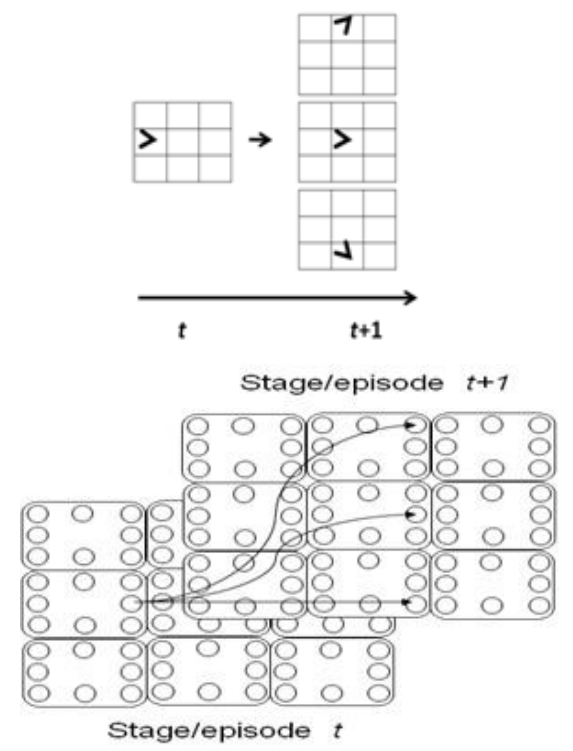

Fig. 3. Agent grid network (directed acyclic graph) excerpt, over consecutive episodes $t$ and $t+1$ for a $3 \times 3$-cell grid. Nodes depict agent state (position, orientation, episode) whereas arcs capture node transition between episodes defined by possible legal moves. Squares refer to grid cells enclosing 8 possible agent orientations. A $|\mathrm{T}|$-move path may be constructed by moving along arcs from stage 0 to stage $|\mathrm{T}|-1$.

Let $\mathcal{G}=(\mathcal{V}, \mathscr{A})$ be the grid network, a directed acyclic graph associated with the agent, where $\mathrm{V}$ $=\bigcup_{t \in T} V_{t}$ is the set of vertices associated to agent states (i.e. position and orientation state variables during a given episode $t \in T=\{1,2, \ldots,|T|-1\})$, and $\mathscr{A}$ the set of arcs $(i, j)$ where $i, j \in V$, reflecting possible agent state transition between consecutive episodes over the grid, corresponding to a legal move $m$ selected from the action set $\mathrm{A}=\{$ left, ahead, right $\}$. $N_{t}=N$ is the set of possible cell locations $\{1, \ldots,|N|\}$ over the grid during episode $t$ whereas $O_{t}=O$ refers to the set of possible agent orientations/headings $\{E, N E, N, N W, W, S W, S, S E\}$ during episode $t$. As a result, $\mathrm{V}=\bigcup_{t \in T}\left(N_{t} \times O_{t}\right)$. The nodes $o$ and $d$ are additional

fictitious origin and destination location vertices defining legal path ends in graph. An excerpt from the abstracted representation for the agent network over two consecutive episodes is given in Fig. 3. An integer binary flow decision variable $x_{i j}$ is associated to each $\operatorname{arc}(i, j) \in \mathscr{A}$. Agent path solution includes $\operatorname{arcs}(i, j) \in \mathscr{A}$ for which $x_{i j}=1$. Given an initial agent state $i_{0}$, path may be defined over the grid network traveling along arcs connecting $o$ to $d$ instantiating flow decision variables to build feasible paths and then, consequently, assigning visit decision variables involved in the objective function. Agent state vertex duplication over $|T|$ episodes is aimed at eliminating disjoint solution subtours otherwise difficult to handle explicitly, and provides a directed acyclic graph to represent a legal solution through binary integer flow decision variables including a multi-cycle path (possible occurrence of many visits on the same cell). Duplication implicitly satisfies path length constraint as well. The significant gain obtained through duplication clearly exceeds the cost incurred by slightly degraded model readability due to the utilization of more complex notations. The agent network includes $|\mathrm{O}||N||T|$ nodes and $|\mathrm{O}||N||T||\mathrm{A}|$ arcs.

\section{B. Mathematical Modeling}

A mathematical mixed-integer linear programming formulation is proposed for the discrete stationary target search and rescue (SAR) path planning problem.

The open-loop decision model captures explicitly ahead of time anticipated information feedback resulting from projected action execution to update the probability (belief) of target cell occupancy. Occupancy belief are assumed to be independent of past search visits as reported in [24]-[26], [5] for the optimal searcher path problem. Accordingly, based on the completion of a projected visit in cell $c$ during time interval $t$, the posterior probability of cell containment $p_{c^{\prime} t+1}$ for any cell $c$ ' is given by:

$$
p_{c^{\prime} t+1}=\left(1-p_{c c} \delta_{c c^{\prime}}\right) p_{c^{\prime} t}
$$

where $\delta_{c c^{\prime}}=1$ if $c^{\prime}=c$ and 0 otherwise. $p_{c^{\prime} t}$ refers to the prior probability/belief of cell $c$ ' target occupancy during time interval $t$ which incorporates "anticipated" information feedback that would result from past visits.

The variables and parameters defining the decision model are given as follows:

$N$ : set of cells defining the grid search area

$T$ : set of time intervals $\{0,1,2, \ldots,|T|-1\}$ defining time horizon.

$V_{c}$ : maximum number of visits on cell $c$

$p_{c c}$ : conditional probability of 'correct' target detection on a visit in cell $c$ given that the target is located in cell $c$.

$\beta_{c}: 1 /\left(1-p_{c c}\right)$

CPOS: cumulative probability of success.

$v_{c l t}$ : cumulative number of visits binary variable. $v_{c l t}=1$ reflects a cumulative number of visits $l$ in cell $c$, by the end of time interval $t$ (otherwise 0 ).

$p_{c t}$ : belief of cell $c$ target occupancy during time interval $t$. $\left\{p_{c 0}\right\}$ refers to the initial belief distribution- target occupancy.

$\operatorname{pos}_{c t \text { : }}$ probability of success (finding the target) as a result of cell $c$ visit at the end of time interval $t$.

$y_{c t}$ : agent position binary variable. $y_{c t}=1$ corresponds to the agent visiting cell $c$ during time interval $t$ (otherwise 0 ).

$x_{i j}$ : state transition binary variable. $x_{i j}=1$ reflects agent network state transition from $i$ to $j$ between episodes.

The MIP decision model may be formulated as follows:

$$
\max C P O S=\max _{\left\{\operatorname{pos}_{c t}\right\}} \sum_{c \in N} \sum_{t \in T} \operatorname{pos}_{c t}
$$

Subject to the linear convex constraint set:

Cell visits

$$
\sum_{0 \leq l \leq V_{c}} v_{c l t}=1 \quad \forall c \in N, \forall t \in T
$$




$$
\sum_{0 \leq l \leq V_{c}} l v_{c l t}=\sum_{0 \leq t^{\prime} \leq t} y_{c t^{\prime}} \quad \forall c \in N, \forall t \in T
$$

Belief update

$$
p_{c t+1}=\sum_{0 \leq l \leq V_{c}} \frac{p_{c 0}}{\beta_{c}^{l}} v_{c l t} \quad \forall c \in N, \forall t \in T
$$

Probability of success

$$
\begin{gathered}
\operatorname{pos}_{c t}-p_{c c} p_{c t} \leq M\left(1-y_{c t}\right) \quad \forall c \in N, \forall t \in T, M>1 \\
\operatorname{pos}_{c t} \leq y_{c t} \quad \forall c \in N, \forall t \in T
\end{gathered}
$$

Initial probability

$$
p_{c 0}=p_{c}(t=0) \quad \forall c \in N
$$

Network coupling

$y_{c t}=\sum_{i_{t}(c) \in \mathbb{V}_{t}} \sum_{j_{t+1} \in \mathbb{V}_{t+1}} x_{i_{t}(c) j_{t+1}} \quad c \in N, t \in T,\left(i_{t}(c), j_{t+1}\right) \in \mathcal{H}$

Initial agent position

$$
\begin{array}{cl}
x_{o i_{0}}=1 & i_{0} \in V \\
y_{c 0}=\delta_{c y_{0}} & \forall c \in N
\end{array}
$$

Initial/final path condition

$$
\begin{gathered}
\sum_{i \in \mathfrak{V}} x_{o i}=1 \\
\sum_{i \in \mathcal{V}} x_{i d}=1
\end{gathered}
$$

Flow conservation

$$
\sum_{i \in \mathbb{V} \cup\{o\}} x_{i j}-\sum_{i \in \mathbb{V} \cup\{d\}} x_{j i}=0 \quad \forall j \in \mathbb{V},(i, j) \in \mathcal{A}
$$

Maximum path length

$$
\sum_{i \in \mathbb{V}} \sum_{j \in \mathbb{V} /\{i\}} x_{i j}=T \quad(i, j) \in \mathcal{H}
$$

Decision variables

$$
\begin{array}{cr}
\operatorname{pos}_{c t}, p_{c t} \in[0,1], y_{c t}, v_{c l t} \in\{0,1\} & c \in N, t \in T \\
x_{i j} \in\{0,1\} \quad(i, j) \in \mathcal{A} & \text { (17) }
\end{array}
$$

The objective function (3) defines cumulative probability of success over the agent path solution and time horizon $|T|$. Constraints are governed through equations (4)-(17). For a given path solution, constraints (4) represent the cumulative number of visits paid on site $c$ by the end of time interval $t$. Constraints (5) simply link that number to past visited locations. The coupling constraints (4) map number of visits and incoming arcs to a site $c$. For area coverage purposes we assume at most $l=V_{c}$ visits to be paid on site $c$. The bound $V_{c}$ can be pre-computed or selected arbitrarily large. Target occupancy probability update is governed by constraint set (6). It is the explicit form of expression (2) relating belief and number of paid visits. Constraint sets (7) and (8) determine the probability of success. Equation (7) reflects a visit requirement to a cell to ensure a feasible observation whereas (8) reinforces null success contributions for alternate unvisited cells. $\mathrm{M}$ is a constant. Initial probability distribution is specified in (9). Constraint sets (10)-(16) reflect model and network coupling as well as flow constraints imposed on/by the agent network. Constraints (10) link cell visits to the agent path network, connecting outgoing arcs from network nodes (states) on stage $t$ to the cell $c$ being visited during episode $t$. Accordingly, arcs $\left(i_{t}(c), j_{t+1}\right)$ relate to any agent state transition starting from position $c$ at stage $t$. Initial agent position and its related network connection are captured in constraints (11)-(12). Constraints (13)-(14) ensure path solution departure and end points to be uniquely defined. Flow conservation governed by constraints (15) aims at balancing the number of incoming and outgoing arcs respectively for a given node. Constraints (16) guarantee a $|T|$-move path solution for an agent, but turn out to be unnecessary as solution constraints are implicitly satisfied by agent network construction.

\section{1) Agent path reconstruction}

On some variable instantiation encoding a computed solution, agent path is reconstructed using the network and its instantiated integer flow decision variables $x_{u v}$. A legal $|T|$-move path is generated by moving along the computed solution arcs from its departure state node $i_{0}$ in stage 0 , up to stage $|T|-1$, before converging to destination node $d$. Algorithm complexity is linear $(O(|T|))$.

\section{COMPUTATIONAL EXPERIMENT}

A computational experiment has been conducted to test the approach for a variety of scenarios. The IBM ILOG CPLEX parallel Optimizer version 12.2.0.0 [22], [23] was used. The barrier method was privileged over the primal-dual technique as it generally explores regions closer to the optimal solution more efficiently. The value of the proposed MIP approach is assessed in terms of optimality gap and run-time, and its performance alternatively compared to a myopic heuristic. Optimality gap is computed as follows:

$$
\text { Opt gap }=\frac{C P O S *-\operatorname{CPOS}_{a}}{C P O S *}
$$

where $C P O S^{*}$ is the optimal cumulative probability of success or a tight upper bound (LP solution), and $C P O S_{a}$ the performance of approach $a$ for a given scenario. The closer (smaller) the optimality gap the better the performance. Simulations were conducted on a 8 core Intel (R) Xeon (R) CPU X5670 $2.93 \mathrm{GHz}$ processor.

\section{A. Myopic Algorithm}

The limited look-ahead method myopically plans moves one step ahead of time, visiting the closest admissible neighbour cell providing the highest gain. At each time step, 
the highest reward move is selected, and the objective function updated accordingly. The procedure is then reiterated for each episode over time horizon $|T|$. Run-time $\in \mathrm{O}(|T|)$.

\section{B. Results}

A sample of random simulation results is reported in Table I for a few 10x10 grid and $T$ horizon scenarios. Each entry corresponds to a separate problem instance. Admissible number of agent moving directions and time horizon scenarios are presented in first and second column respectively. Cumulative probability of success $(C P O S)$ and optimality gap for the myopic method and the optimal CPLEX solver refer to the third and fourth column. Run-time measured in seconds is given in the last column.

TABLE I: Relative PERFoRmanCE OF CPLEX SOLVER (MIP) VS MyopiC HEURISTIC FOR A SAMPLE DATA SET (10X10 GRID)

\begin{tabular}{|c|c|c|c|c|c|c|}
\hline \multirow{2}{*}{$\begin{array}{c}\# \\
\text { Agent } \\
\text { Moves } \\
\text { /Inst. }\end{array}$} & \multirow{2}{*}{$\begin{array}{c}\text { Time } \\
\text { Horizon } \\
|T|\end{array}$} & \multicolumn{2}{|c|}{$\begin{array}{c}\text { Myopic } \\
\text { Heuristic }\end{array}$} & \multicolumn{2}{|c|}{$\begin{array}{c}\text { CPLEX } \\
\text { Solver - MIP }\end{array}$} & \multirow{2}{*}{$\begin{array}{c}\text { CPLEX } \\
\text { Solver } \\
\text { Run-time } \\
(s) \\
\end{array}$} \\
\hline & & CPOS & $\begin{array}{r}\text { Opt } \\
\text { gap \% }\end{array}$ & CPOS & $\begin{array}{l}\text { Opt } \\
\text { tp \% }\end{array}$ & \\
\hline $3-$ & 20 & 0.239 & 30.94 & 0.346 & 0 & 1.81 \\
\hline $3 \mathrm{~A}$ & 20 & 0.186 & 42.8 & 0.325 & 0 & 1.67 \\
\hline $3 \mathrm{~B}$ & 20 & 0.288 & 16.7 & 0.346 & 0 & 1.83 \\
\hline $3 \mathrm{C}$ & 20 & 0.202 & 37.0 & 0.321 & 0 & 2.03 \\
\hline $3 \mathrm{D}$ & 20 & 0.288 & 16.7 & 0.345 & 0 & 1.80 \\
\hline $3 \mathrm{E}$ & 20 & 0.185 & 60.3 & 0.465 & 0 & 5.12 \\
\hline $3 \mathrm{~F}$ & 20 & 0.432 & 28.9 & 0.608 & 0 & 2.95 \\
\hline $3 \mathrm{G}$ & 12 & 0.321 & 18.9 & 0.396 & 0 & 0.14 \\
\hline $3 \mathrm{H}$ & 12 & 0.284 & 27.6 & 0.392 & 0 & 0.16 \\
\hline $3 \mathrm{I}$ & 12 & 0.229 & 49.8 & 0.456 & 0 & 0.17 \\
\hline $3 \mathrm{~J}$ & 20 & 0.198 & 32.6 & 0.293 & 0 & 2.50 \\
\hline
\end{tabular}

Results show that an optimal solution may be computed on a second timescale. The fast MIP solver may in some cases outperform the myopic procedure by a large margin (e.g. more than 50\%). Providing the best solution and measurable gain (upper bound through Lagrangean integrality constraint relaxation) over alternate heuristics for practical size problems for the first time, the approach may be repeatedly reused in dynamic settings exploiting intermediate sensor readings, given its small run-time.

A simulation run for a $T=40$ scenario even reported computation of an optimal solution in 150 seconds which is remarkably efficient. Faster hardware and additional processing elements might further extend computable $T$.

\section{CONCLUSION}

An innovative mixed-integer linear programming (MIP) approach has been proposed to solve a probabilistic open-loop search and rescue path planning problem with anticipated feedback. Small computational cost naturally allows dynamic planning through a closed-loop environment settings where real information feedback resulting from past sensor agent observations is exploited to compute a revisited solution over a rolling horizon during the next cycle (episode). The novelty of the approach lies in the combination of a new problem formulation, an original network representation, and a problem-solving procedure based on linear programming CPLEX technology to efficiently compute optimal solution for practical size problems, usually handled through heuristic methods. For the first time, an upper bound estimate of the best solution naturally derived from the approach may be used for convergence or performance comparison analysis purposes, and/or trading-off solution quality and execution time. Experimental results demonstrate the value of the proposed approach for practical size problems.

\section{REFERENCES}

[1] K. E. Trummel and J. R Weisinger, "The complexity of the optimal searcher path problem," Operations Research, vol. 34, no. 2, pp. 324-327, 1986.

[2] S. Benkoski, M. Monticino, and J. Weisinger, "A survey of the search theory literature," Naval Research Logistics, vol. 38, pp. 469-494, 1991.

[3] L. Stone, "What's happened in search theory since the 1975 Lanchester Prize?" Operations Research, vol. 37, no. 3, 1989.

[4] R. Hohzaki and K. Iida, "Optimal search plan for a moving target when a search path is given," Math. Japonica, vol. 41, no. 1, pp. 175-184, 1995.

[5] H. Lau, "Optimal Search in Structured Environments," Ph.D. dissertation, University of Technology, Sydney, Australia, 2007.

[6] H. Lau and G. Dissanayake, "Probabilistic search for a moving target in an indoor environment," in Proc. IEEE/RSJ Int. Conf. Intelligent Robots and Systems, 2006, pp. 3393-3398.

[7] H. Lau and G. Dissanayake, "Optimal search for multiple targets in a built environment," in Proc. IEEE/RSJ Int. Conf. Intelligent Robots and Systems, Edmonton, Alberta, Canada, 2005, pp. 228-233.

[8] J.-C. Latombe, "Robot Motion Planning," International Series in Engineering and Computer Science; Robotics: Vision, Manipulation and Sensors, Boston, Kluwer Academic Publishers, vol. 124, 1991.

[9] C. Choo, J. Smith, and N. Nasrabadi, "An efficient terrain acquisition algorithm for a mobile robot," in Proc. IEEE Int. Conf. Robotics and Automation, Sacramento, CA, 1991, pp. 306-311.

[10] A. Sankaranarayanan and I. Masuda, "Sensor based terrain acquisition: A new, hierarchical algorithm and a basic theory," in Proc. IEEE/RSJ Int. Conf. Intelligent Robots and Systems, Raleigh, 1992, pp. 1515-1523.

[11] J. Svennebring and S. Koenig, "Building terrain-covering ant robots: A feasibility study," Auton. Robots, vol. 16, no. 3, pp. 313-332, 2004.

[12] S. Wong and B. MacDonald, "A topological coverage algorithm for mobile robots," in Proc. IEEE/RSJ Int. Conf. Intelligent Robots and Systems, Las Vegas, 2003, pp. 1685-1690.

[13] S. Yang and C. Luo, "A neural network approach to complete coverage path planning," IEEE Trans. Syst.,Man, Cybern. B, vol. 34, no. 1, pp. 718-724, 2004.

[14] I. Rekleitis et al., "Efficient boustrophedon multi-robot coverage: an algorithmic approach," Ann Math Artif. Intell., vol. 52, pp. 109-142, 2008.

[15] W. Wagner et al., "Distributed covering by ant-robots using evaporating traces," IEEE Trans. Robot. Autom., vol. 15, no. 5, pp. 918-933, 1999.

[16] J. O. Royset and H. Sato, "Route Optimization for Multiple Searchers," Naval Research Logistics, vol. 57, no. 8, pp. 701-717, 2010.

[17] T. H. Chung and J. Burdick, "Analysis of search decision making using probabilistic search strategies," IEEE Transactions on Robotics, vol. 28, no. 10, pp. 132-144, 2012.

[18] Y. Jin, Y. Liao, A. Minai, and M. Polycarpou, "Balancing search and target response in cooperative unmanned aerial vehicle (UAV) Teams," IEEE Trans on Sys Man and Cybern. Part B, vol. 36, no. 3, pp. 571-587, 2006.

[19] G. A. Hollinger, "Search in the Physical World," Ph.D. dissertation, CMU-RI-TR-10-20, Robotics Institute, Carnegie Mellon University, 2010.

[20] T. Chung, "On Probabilistic Search Decisions under Searcher Motion Constraints," Workshop on Algorithmic Foundation of Robotics VIII, Guanajuato, Mexico, pp. 501-516, 2009.

[21] G. A. Hollinger and S. Singh, "GSST: Anytime Guaranteed Search with Spanning Trees," Autonomous Robots, vol. 29, no. 1, pp. 99-118, 2010.

[22] IBM ILOG CPLEX Optimizer. [Online]. Available: http://www-01.ibm.com/software/integration/optimization/cplex-opti mization-studio/cplex-optimizer/cplex-performance/ 
[23] IBM ILOG CPLEX. (2009). [Online]. 12(1). Available: ftp://public.dhe.ibm.com/software/websphere/ilog/docs/optimization/c plex/ps_usrmancplex.pdf

[24] M. Morin, A. P. Papillon, F. Laviolette, I. Abi-Zeid, and C. G. Quimper, "Constraint Programming for Path Planning with Uncertainty: Solving the Optimal Search Path problem," in Proc. the 18th Conference on Principles and Practice of Constraint Programming, Québec, Qc, Canada, 2012, pp. 988-1003.

[25] J. N. Eagle and J. R. Yee, "An optimal branch and bound procedure for the constrained path, moving target search problem," Operations Research, vol. 38, no. 1, pp. 110-114, 1990.

[26] R. F. Dell, J. N. Eagle, G. H. A. Martins, and A. G. Santos, "Using multiple searchers in constrained-path, moving-target search problems," Naval Research Logistics, vol. 43, pp. 463-480, 1996.

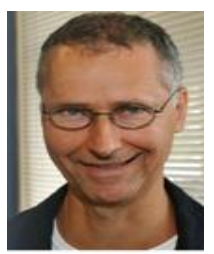

Jean Berger is a Defence Scientist with the Command, Control and Intelligence Section of Defence Research and Development Canada - Valcartier, working in the field of information technology. He received BS and MS degrees in engineering physics from Ecole Polytechnique de Montréal, Canada. His research interests include artificial intelligence and operations research applied to intelligent control, planning, surveillance and reconnaissance, and logistics.

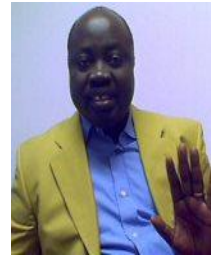

Nassirou Lo is the Chairman \& CEO of the company T-OPTLOGIC Inc. and a lecturer in the École des sciences de l'administration TÉLUQ, Université du Québec Department of Labour, Economics and Management at Téluq. He received a Ph.D. and a MBA in pure mathematics from Université des Sciences et Technologies de LILLE I (U.S.T.L.), in France. His research interests include all branch of pure and applied mathematics. He has been working in the area of operations research and management for the past ten years.

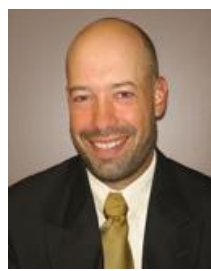

Martin Noel is director of academic and research at Téluq, université du Québec. He has taught courses in operations research, statistics and operations management. He earned a Ph.D. in management science and a MBA in operations management from Laval University. He also received a B.Sc. degree in statistics from the University of Western Ontario. His research interests include stochastic and network modeling applied to control and planning problems in tool management, path planning and logistics. 